\title{
Terapia ocupacional assistida por cáes: uma revisão de escopo da literatura brasileira
}

\section{Canine-assisted Occupational Therapy: a scoping review of the brazilian literature}

\author{
Mirela de Oliveira Figueiredo ${ }^{\mathrm{a}}$ (D), Ana Luiza Alegretti ${ }^{\mathrm{b}}$ (D), Lilian Magalhães ${ }^{\mathrm{a}}$ (D) \\ ${ }^{a}$ Universidade Federal de São Carlos - UFSCar, São Carlos, SP, Brasil. \\ bUniversity of Texas Health Science Center, San Antonio, Texas, USA.
}

Como citar: Figueiredo, M. O., Alegretti, A. L., \& Magalhães, L. (2021). Terapia ocupacional assistida por cães: uma revisão de escopo da literatura brasileira. Cadernos Brasileiros de Terapia Ocupacional, 29, e2087. https://doi.org/10.1590/2526-8910.ctoAR2087

\begin{abstract}
$\underline{\text { Resumo }}$
Terapeutas ocupacionais têm praticado a Terapia Assistida por Animais (TAA) incorporando cães em suas intervenções. A produção de conhecimento nacional sobre a Terapia Ocupacional Assistida por Cáes foi analisada por meio de uma revisão de escopo. Esta investigação buscou obter respostas sobre quais populaçóes têm sido focalizadas na Terapia Ocupacional Assistida por Cáes no Brasil, quais os objetivos e resultados terapêuticos, de que forma o cão atuou neste processo, qual o treinamento necessário aos cães e qual a formação requerida ao terapeuta ocupacional para realizar tal terapia. A busca ocorreu nos periódicos nacionais de Terapia Ocupacional e na biblioteca Scielo.br por meio de descritores e critérios de inclusão. Não foram encontrados estudos da terapia ocupacional tendo cães como assistentes, mas sim sobre o cuidar de animais como uma ocupação humana e um papel ocupacional $(\mathrm{n}=4)$. Um estudo referiu a terapia ocupacional como uma das profissóes que, no contexto estrangeiro, realiza a terapia assistida por cães com pessoas com deficiência e/ou sequelas físicas ou mentais, sendo os objetivos e resultados terapêuticos de reabilitação física ou cognitiva. A revisão revela que a produção nacional é incipiente, sobretudo se comparada à literatura estrangeira, que relata sobre a Terapia Ocupacional Assistida por Cães e informa sobre as populaçóes, objetivos e resultados terapêuticos, a formação e competências necessárias ao terapeuta e o treinamento requerido ao cáo. Reafirma-se a necessidade de estudos e de produção de conhecimento nacional para o embasamento teórico e diretrizes para a prática da Terapia Ocupacional Assistida por Cães no Brasil.
\end{abstract}

Palavras-chave: Terapia Assistida por Animais, Terapia Ocupacional, Cáes, Terapia Assistida por Cães, Literatura de Revisão como Assunto.

\section{$\underline{\text { Abstract }}$}

Occupational therapists have practiced Animal-Assisted Therapy (AAT) by incorporating dogs in their interventions. The production of national knowledge 
about Canine-assisted Occupational Therapy was analyzed through a scoping review. This investigation sought to obtain answers about which populations have been focused on Canine-assisted Occupational Therapy in Brazil, what are the objectives and therapeutic results, how the dog acted in this process, what training is necessary for the dogs and what training is required for the occupational therapist to carry out such therapy. The research occurred in the national journals of Occupational Therapy and Scielo.br library through descriptors and inclusion criteria. No studies of occupational therapy were found with dogs as assistants, but on the care of animals composing a human occupation and an occupational role $(\mathrm{n}=4)$. One study mentioned occupational therapy as one of the professions that, in the international context, perform Canine-assisted Occupational Therapy with people with disabilities and/or physical or mental sequelae, being the therapeutic objectives and results of physical or cognitive rehabilitation. The review reveals that national production is incipient, especially compared to the international literature that reports on Canine-assisted Occupational Therapy and informs about populations, therapeutic objectives, and results, the training, and skills needed by the therapist, and the training required for the dog. The need for studies and the production of national knowledge for the theoretical basis and guidelines for the practice of Canine-assisted Occupational Therapy in Brazil is reaffirmed.

Keywords: Animal Assisted Therapy, Occupational Therapy, Dogs, Canineassisted Therapy, Literature Review as Topic.

\section{Introduçáo}

A Terapia Assistida por Animais (TAA) data da década de 1960, nos Estados Unidos, com base no trabalho de Boris Levinson, que incluiu seu próprio cão na terapia com crianças. Na década de 1970, o procedimento foi continuado por Samuel Corson e Elisabeth Corson, que o denominaram de "pet therapy". O modelo continuou a se desenvolver por todo o mundo, com a composição de diferentes áreas e campos de conhecimento, o que garantiu a ampliação dos seus princípios, fundamentos e terminologia. Entretanto, esta amplitude terminológica, por vezes, tem fornecido subsídios parciais e dificultado uma compreensão consensual (Fine et al., 2019).

LaJoie (2003), em revisão de literatura, encontrou 20 definições diferentes para a TAA, além de 12 termos diferentes para o mesmo fenômeno. Por isso, para padronização terminológica, foi instaurado o termo Intervençóes Assistidas por Animais (IAA), sendo classificado e diferenciado em três tipos: Terapia Assistida por Animais (TAA), Atividade Assistida por Animais (AAA) e Educação Assistida por Animais (EAA) (Kruger \& Serpell, 2006).

A Terapia Assistida por Animais (TAA) consiste na incorporação de um animal como parte integrante do processo terapêutico, sendo que as intervençôes são organizadas e supervisionadas por um profissional da área da saúde que possui objetivos previamente determinados, sendo avaliados e registrados os resultados das intervençôes. A Atividade Assistida por Animais (AAA) visa a melhorar a qualidade de vida dos assistidos, não requer a supervisão de um profissional da saúde e os resultados não serão necessariamente avaliados. Já a Educação Assistida por Animais (EAA) consiste em um 
recurso pedagógico em que o animal é considerado parte integrante do processo de ensino e aprendizagem formal ou informal ou do processo de socializaçáo na vida escolar (Friedmann et al., 2015a; Reed et al., 2012).

Os primeiros estudos internacionais sobre os benefícios das TAA também datam da década de 60, com pacientes que apresentavam quadros clínicos psiquiátricos (Squilasse \& Squilasse Junior, 2018). Com o passar dos anos, houve um significativo desenvolvimento teórico sobre as abordagens que incluem os animais em contextos de tratamento, ampliando a população atendida e os objetivos (Şahin et al., 2018), como com crianças com paralisia cerebral, distúrbios neurológicos do desenvolvimento, distúrbios do espectro autista, distúrbios do processamento sensorial, neurológicos degenerativos e/ou musculares com foco, por exemplo, no controle do tônus muscular, mobilidade e equilíbrio (Martin \& Farnum, 2002).

Atualmente, há uma quantidade expressiva de serviços de saúde nos países industrializados que concretizam a TAA em centros de reabilitação física e/ou psiquiátricos. Com isso, a população atendida pela TAA tem sido diversa, abrangendo diferentes faixas etárias e acometimentos com a finalidade de melhorar estados emocionais e psicossociais, habilidades físicas, sensoriais e/ou cognitivas (Şahin et al., 2018).

Um dos animais frequentemente utilizados na TAA é o cão, sendo este preferido pelos profissionais por ser considerado amigável, simpático, obediente, brincalhão e com melhor e imediata interação com as pessoas. Com isso, os cães são considerados com grande potencial para melhorar as habilidades físicas, cognitivas, funcionais e sociais dos indivíduos, para aumentar o grau de independência e autonomia na realização das atividades de vida diária e para facilitação da comunicação e interação com humanos (Şahin et al., 2018).

Nas últimas décadas, pesquisas têm demonstrado o potencial dos cães nos programas de reabilitação, para a saúde, bem-estar e qualidade de vida de pessoas com diferentes idades e acometimentos mentais, físicos e/ou cognitivos (Hall et al., 2017). Estudos identificaram a redução do estresse em crianças (Nagengast et al., 1997; Hansen et al., 1999; Viau et al., 2010), melhora na saturação de oxigênio de pacientes com câncer em quimioterapia (Orlandi et al., 2007), redução da variabilidade da frequência cardíaca em adultos (Motooka et al., 2006), redução da pressão arterial diastólica (Tsai et al., 2010), reduções nos níveis de epinefrina e noradrenalina, pressão sistólica da artéria pulmonar e pressão da cunha capilar pulmonar em pacientes com insuficiência cardíaca (Cole et al., 2007), diminuição do cortisol sérico nos profissionais de saúde (Barker et al., 2005), melhora na qualidade de vida de residentes em casas de repouso com demência (Nordgren \& Engstrom, 2012) e de familiares de crianças com síndrome de down e outras com graves deficiências físicas e mentais (Stumpf \& Breitenbach, 2014), aumento da atividade física, melhora da função física e da qualidade de vida em idosos (Abate et al., 2011; Friedmann et al., 2015b), melhora no desempenho da vida diária e das habilidades de caminhada de mulheres residentes em casas de repouso por demência (Nordgren \& Engstrom, 2012), diminuição da dor em crianças hospitalizadas (Braun et al., 2009).

No contexto internacional, terapeutas ocupacionais praticam a TAA incorporando cães em suas intervenções voltadas para distintas populaçôes e objetivos terapêuticos (Camp, 2001; Fairman \& Huebner, 2001; Velde et al., 2005; Isaacson, 2013; VanFleet et al., 2019; Herlache-Pretzer et al., 2017). Alguns terapeutas ocupacionais e pesquisadores têm enfocado no impacto de cães de assistência (cães de serviço, do inglês 
service dog) para o desenvolvimento de crianças, adolescentes e adultos e para o estabelecimento de relaçóes saudáveis entre familiares e filhos com diversos acometimentos de saúde (Viau et al., 2010; Davis et al., 2004; Smyth \& Slevin, 2010).

No contexto nacional, há registros de que, na década de 50, Nise da Silveira, médica psiquiatra e responsável pelo setor de Terapia Ocupacional do Centro Psiquiátrico D. Pedro II, localizado no Rio de Janeiro, incluía animais no tratamento de pacientes esquizofrênicos. A Dra. Nise da Silveira observou uma vinculação que surgia entre os pacientes e os cães, sendo que o cão se tornava um ponto de referência no mundo externo, o que facilitava a retomada do contato do paciente com a realidade, e por isso ela os denominou de co-terapeutas (Squilasse \& Squilasse Junior, 2018).

Levando em consideração as comprovaçóes científicas em âmbito internacional sobre a eficácia dos cães nas TAA e nas intervençóes da terapia ocupacional, o presente estudo objetivou investigar a produçâo de conhecimento da Terapia Ocupacional brasileira sobre a Terapia Assistida por Animais, especificamente sobre a Terapia Ocupacional Assistida por Cães.

\section{Método}

Neste estudo, realizou-se uma revisão de escopo. Este tipo de revisão estabelece o mapeamento das fontes e tipos de evidências disponíveis que subsidiam uma área de pesquisa (Arksey \& O’Malley, 2005). Trata-se de um procedimento transversal que possibilita a identificaçáo dos conceitos e das lacunas existentes em uma área de pesquisa. É, preferencialmente, aplicada a áreas que ainda não tenham sido revisadas de forma abrangente anteriormente (Arksey \& O’Malley, 2005), como é o caso do presente estudo.

Os parâmetros recomendados por Arksey \& O’Malley (2005) e retrabalhados por O’Brien et al. (2016), Peters et al. (2015), Colquhoun et al. (2014) e Tricco et al. (2016) foram adotados neste estudo, que foi realizado em 5 etapas:

1) definição das perguntas de pesquisa;

2) identificação dos estudos relevantes por meio de diferentes fontes;

3) composição da amostra final com base nos critérios de busca e inclusão/exclusão;

4) extração dos dados relacionados à pergunta de pesquisa, incluindo informaçóes gerais sobre o estudo;

5) descrição dos dados, análise numérica e temática/conceitual dos dados, discussão.

As perguntas investigativas que conduziram este estudo foram: Que populaçóes têm sido focalizadas na Terapia Ocupacional que realiza a Terapia Assistida por Cáes no Brasil? Há indicaçóes sobre os objetivos e resultados terapêuticos alcançados, bem como de que forma o cão atuou para auxiliar neste processo no Brasil? Como tem sido referido o treinamento que os cães recebem para se tornarem assistentes nas sessóes de terapia ocupacional no Brasil? Como tem sido referida a formação que o terapeuta ocupacional necessita para realizar a Terapia Ocupacional Assistida por Cães no Brasil?

\section{Procedimentos de Coleta e Análise dos Dados}

Foram analisados os periódicos específicos da área Cadernos Brasileiros de Terapia Ocupacional da UFSCar (CaBTO), Revista de Terapia Ocupacional da USP (Revista 
TO-USP), Revista Baiana de Terapia Ocupacional e Revista Interinstitucional Brasileira de Terapia Ocupacional (RevisBrato), bem como a biblioteca digital Scielo.br, pois esta possui uma coleção de periódicos científicos brasileiros nos quais os estudos da terapia ocupacional brasileira são publicados. Foram aplicados os descritores em Ciências da Saúde contidos no Descritores em Ciências da Saúde (DeCS): "terapia assistida por animais" e os sinônimos "mascote terapia”, "mascote-terapia”, "terapia facilitada por animais de estimação", "terapia com animais de estimação" e "uso terapêutico de animais de estimação" em combinação com "terapia ocupacional"

Para a composição da amostra, foram consideradas publicações: a) disponíveis online nos periódicos e no Scielo.br, independentemente dos objetivos, população ou metodologia do estudo; b) produzidas por terapeutas ocupacionais sobre a TAA, especificamente tendo os cáes como assistentes; c) sobre fundamentos da prática da Terapia Ocupacional que subsidiassem a prática da Terapia Assistida por Cáes; d) sobre a Terapia Assistida por Animais que se referiam à terapia ocupacional ou a mencionavam.

Com isso, foram excluídos artigos que, apesar de aparecerem na busca, não continham os descritores no texto ou, apesar de conterem os descritores no título, resumo ou no texto, não foram produzidos por terapeutas ocupacionais e/ou náo mencionaram a terapia ocupacional, não fornecendo subsídios para a prática da TAA por terapeutas ocupacionais.

Foram consideradas apenas as publicações disponíveis online, por isso, o período analisado nos Cadernos Brasileiros de Terapia Ocupacional da UFSCar foi de 1990 a 2020, na Revista de Terapia Ocupacional da USP, de 2002 a 2020, na Revista Baiana de Terapia Ocupacional, de 2005 a 2020, na RevisBRATO, de 2013 a 2020, no Scielo.br não foram usados parâmetros temporais.

Com a utilização dos descritores anteriormente referidos, não foram encontrados artigos nos Cadernos Brasileiros de Terapia Ocupacional, na Revista Baiana de Terapia Ocupacional e na RevisBRATO. Foram identificados 22 artigos na Revista de Terapia Ocupacional da USP e 6 artigos no Scielo.br.

$\mathrm{Na}$ Revista de Terapia Ocupacional da USP, os artigos que apareceram com os descritores "terapia assistida por animais" $(n=3)$, "terapia facilitada por animais de estimação" ( $n=1)$, "terapia com animais de estimação" ( $n=5)$ e "uso terapêutico de animais de estimação" ( $\mathrm{n}=2)$ foram os mesmos que apareceram quando foi associado cada descritor à "terapia ocupacional". Portanto, os artigos encontrados foram computados apenas uma vez, totalizando 11 artigos. Ao revisar os títulos desses 11 artigos, foram verificadas repetiçóes. Os 2 artigos encontrados com o descritor "uso terapêutico de animais de estimação", o único artigo encontrado com o descritor "terapia facilitada por animais de estimação" e um dos artigos encontrados com o descritor "terapia com animais de estimação" duplicavam os encontrados com o

\footnotetext{
${ }^{1}$ Importante esclarecer que os sinônimos encontrados no DeCs para o termo "terapia assistida por animais" não são recomendados pelas organizaçóes internacionais voltadas à interação homem-animal e intervenção assistida por animais (International Association of HumanAnimal Interaction Organizations, 2018). Entretanto, estes termos contidos no DeCS foram adotados para a coleta de dados uma vez que servem como fonte "[...] de terminologia comum para pesquisa em múltiplos idiomas, proporcionando um meio consistente e único para a recuperaçāo da informação" (Centro Latino-Americano e do Caribe de Informação em Ciências da Saúde, 2018). Os termos “pet terapia” e "pet therapy" não foram adotados nesta revisão, uma vez que não constam no DeCs e por serem considerados uma terminologia em desuso desde o surgimento e adoção do termo Intervenção Assistida por Animais, bem como sua classificação em três tipos Terapia Assistida por Animais, Atividade Assistida por Animais e Educação Assistida por Animais, por estudiosos, praticantes e associaçôes voltadas para essas intervençốes (Fine et al., 2019).
} 
descritor "terapia assistida por animais". Desta forma, foram considerados para análise os 3 artigos encontrados com o descritor "terapia assistida por animais" e 4 artigos encontrados com o descritor "terapia com animais de estimação", totalizando 7 artigos. Destes 7 artigos, 3 foram excluídos por não atenderem aos critérios já mencionados.

Dentre os 6 artigos encontrados no Scielo.br, com base no descritor "terapia assistida por animais", apenas 1 mencionava a terapia ocupacional. A amostra final foi composta por 5 estudos, sendo 4 da Revista de Terapia Ocupacional da USP e 1 do Scielo.br. Na Figura 1, ilustra-se o processo de busca com a identificação dos artigos, exclusão das duplicidades, exclusão por não cumprimento dos critérios de elegibilidade e composição da amostra final.

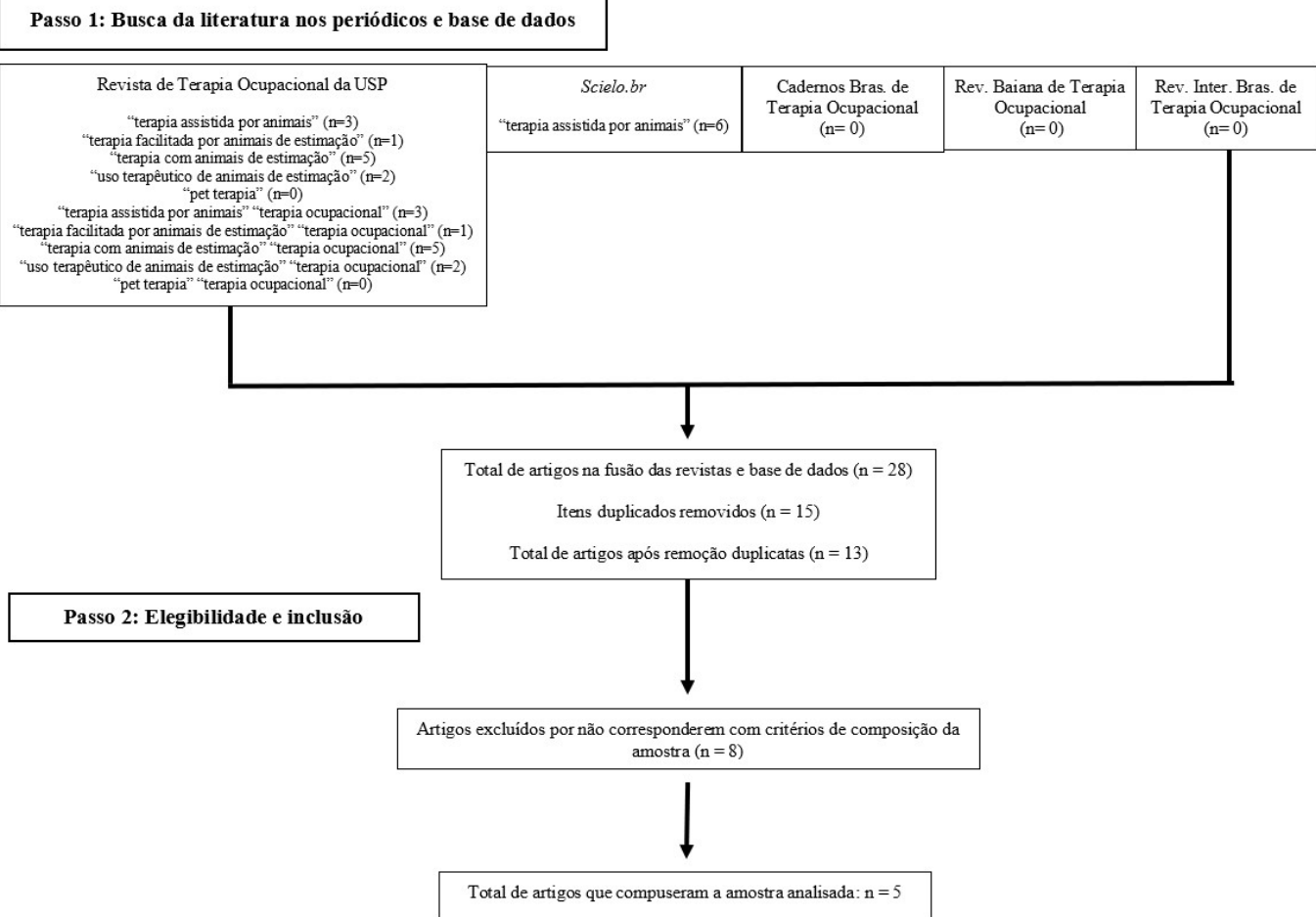

Figura 1. Fluxograma do Processo de Busca e Composição da Amostra. Fonte: Elaborado pelos autores.

Foram utilizados três formulários para registro das informaçôes. O primeiro dirigido à organização de todos os artigos que apareceram na busca com os descritores, outro para o armazenamento dos artigos excluídos por repetição ou por não cumprirem os critérios, e um terceiro para a organização das informaçóes dos artigos que compuseram a amostra final, referentes à identificaçáo do estudo (autor/ano, objetivos do estudo, tipo do estudo, amostra) e conteúdos relacionados às perguntas de pesquisa.

A primeira fase da análise dos dados foi quantitativa, com o somatório dos artigos encontrados e composição da amostra final, somada à análise simples de frequência. A segunda fase da análise foi qualitativa, envolvendo a leitura de todos os artigos na íntegra e a extração de informaçóes que respondessem às perguntas investigativas. 


\section{Resultados}

Os 5 estudos analisados foram publicados entre 2014 e 2019, sendo dois estudos teóricos, dois qualitativos e um de revisão sistemática. Os estudos com participantes $(\mathrm{n}=3)$ envolveram crianças, adolescentes e seus familiares. Os estudos possuíam objetivos diferentes e apenas nos respectivos resultados foi possível extrair dados que respondessem às perguntas que orientaram esta revisão de escopo. Não foi encontrado, nos periódicos e na base analisada, nenhum estudo produzido por terapeutas ocupacionais sobre a Terapia Assistida por Animais tendo o cáo como assistente.

Foram encontradas 2 publicaçóes, o documento da Associação Americana de Terapia Ocupacional (American Occupational Therapy Association, 2015) e o artigo de Dias et al. (2014), que mencionava o ato de cuidar dos animais enquanto parte das atividades cotidianas dos seres humanos compondo a dimensão ocupacional do cuidado de si, de outros e do ambiente em que se vive.

No documento da Associação Americana de Terapia Ocupacional, denominado "Estrutura da Prática da Terapia Ocupacional: Domínio e Processo" (American Occupational Therapy Association, 2015), define-se que o cuidar de animais se insere nas Atividades Instrumentais de Vida Diária (AIVDs). Esses atos são descritos como o de "[...] organizar, supervisionar ou prestar cuidados a animais de estimação e animais de serviço" (American Occupational Therapy Association, 2015, p. 20). Nesse tópico, o documento também faz referência aos animais como componentes do ambiente físico das pessoas, ou seja, "[...] o ambiente não-humano natural ou construído, que cerca as pessoas e nos quais suas ocupaçôes se concretizam" (American Occupational Therapy Association, 2015, p. 29).

Na mesma direção, o estudo de Dias et al. (2014) refere o "cuidar de animais" compondo as atividades de lazer/passatempo frequentemente realizadas por idosos, sendo considerado uma Atividade Avançada de Vida Diária (AAVD).

Outros 2 estudos encontrados, de Cid et al. (2017) e Lopes \& Matsukura (2018), referiram-se ao desejo das pessoas em processo de tratamento de estarem perto de seus animais de estimação e de poderem cuidar destes. Cid et al. (2017) indicaram que o cuidar de animais foi caracterizado enquanto uma atividade que compóe a rotina e propicia o senso de responsabilidade da criança em sofrimento psíquico. Para Lopes $\&$ Matsukura (2018), o estar perto dos animais de estimação constituía um dos desejos de adolescentes com paralisia cerebral.

Foi encontrado um estudo sobre TAA que mencionou a terapia ocupacional enquanto uma das áreas profissionais de conhecimento que realiza a TAA. O estudo de Mandrá et al. (2019), de revisão sistemática, localizou apenas dois estudos que envolviam a terapia ocupacional com cães. Esses estudos localizados foram realizados na América do Norte e fornecem informaçóes sobre a população assistida (pessoas com deficiência física ou mental, ou sequelas de demência, ou de paralisia cerebral). Segundo os autores, os objetivos terapêuticos dos estudos abrangiam a reabilitação física ou cognitiva, sendo indicado que foram alcançados. Entretanto, Mandrá et al. (2019) referem que não fica clara a forma como o cão atuou para auxiliar neste processo.

$\mathrm{Na}$ Tabela 1, detalham-se as informaçôes registradas sobre as publicaçóes que compuseram a amostra analisada. 
Tabela 1. Descrição dos artigos analisados.

\begin{tabular}{|c|c|c|c|c|}
\hline Autor/Ano & Objetivo do Estudo & Tipo de Estudo & Amostra & $\begin{array}{l}\text { Dados Relacionados às } \\
\text { Perguntas de Pesquisa }\end{array}$ \\
\hline Dias et al. (2014) & $\begin{array}{c}\text { Discutir sobre as principais } \\
\text { características das atividades avançadas } \\
\text { de vida diária (AAVD), sua classificação } \\
\text { em domínios de atividades social, físico, } \\
\text { produtivo e de lazer e ressalta a } \\
\text { importância da inclusão das AAVD na } \\
\text { avaliaçáo funcional dos idosos. }\end{array}$ & Teórico & Textual & $\begin{array}{l}\text { o “cuidar de animais" consiste } \\
\text { em uma das atividades de } \\
\text { lazer/passatempo de idosos. }\end{array}$ \\
\hline $\begin{array}{c}\text { American } \\
\text { Occupational } \\
\text { Therapy } \\
\text { Association (2015) }\end{array}$ & $\begin{array}{c}\text { Divulgar a traduçáo do documento } \\
\text { oficial da Associaçáo Americana de } \\
\text { Terapia Ocupacional (AOTA): “A } \\
\text { Estrutura da Prática da Terapia } \\
\text { Ocupacional: Domínio e Processo, 3a } \\
\text { Edição”. }\end{array}$ & Teórico & Textual & $\begin{array}{l}\text { o “cuidar de animais" é } \\
\text { considerado uma das Atividades } \\
\text { Instrumentais de Vida Diária } \\
\text { (AIVDs), sendo que os animais } \\
\text { compōem o ambiente em que } \\
\text { as pessoas concretizam suas } \\
\text { ocupaçóes. }\end{array}$ \\
\hline Cid et al. (2017) & $\begin{array}{c}\text { Identificar as percepçóes de } \\
\text { responsáveis por crianças em } \\
\text { sofrimento psíquico sobre as atividades } \\
\text { que desenvolvem com elas, as práticas } \\
\text { educativas que utilizam em seu } \\
\text { cotidiano e sobre como compreendem } \\
\text { a influência destas no comportamento } \\
\text { das crianças. }\end{array}$ & $\begin{array}{c}\text { Estudo } \\
\text { qualitativo }\end{array}$ & $\begin{array}{l}\text { Criança em } \\
\text { sofrimento } \\
\text { psíquico e } \\
\text { familiares }\end{array}$ & $\begin{array}{l}\text { o cuidar de animais compóe a } \\
\text { rotina e propicia o senso de } \\
\text { responsabilidade da criança em } \\
\text { sofrimento psíquico. }\end{array}$ \\
\hline $\begin{array}{c}\text { Lopes } \& \\
\text { Matsukura (2018) }\end{array}$ & $\begin{array}{l}\text { Conhecer as perspectivas em relação à } \\
\text { participação social de adolescentes com } \\
\text { Paralisia Cerebral, sob a ótica dos } \\
\text { próprios adolescentes e de seus } \\
\text { familiares. }\end{array}$ & $\begin{array}{c}\text { Estudo } \\
\text { qualitativo }\end{array}$ & $\begin{array}{l}\text { Adolescente com } \\
\text { paralisia cerebral e } \\
\text { familiares }\end{array}$ & $\begin{array}{l}\text { estar perto dos animais de } \\
\text { estimaçáo constitui um dos } \\
\text { desejos dos adolescentes com } \\
\text { paralisia cerebral. }\end{array}$ \\
\hline $\begin{array}{l}\text { Mandrá et al. } \\
\text { (2019) }\end{array}$ & $\begin{array}{l}\text { Verificar evidências sobre aplicação da } \\
\text { Terapia Assistida por Animais na saúde } \\
\text { realizando revisáo sistemática da } \\
\text { literatura. }\end{array}$ & $\begin{array}{c}\text { Revisão } \\
\text { sistemática }\end{array}$ & $\begin{array}{l}\text { Artigos das bases } \\
\text { de dados: } \\
\text { MedLine, } \\
\text { PubMed, Scopus, } \\
\text { Lilacs Scielo }\end{array}$ & $\begin{array}{c}\text { a terapia ocupacional foi } \\
\text { identificada enquanto uma das } \\
\text { áreas profissionais que realizou } \\
\text { TAA, sendo especificamente } 2 \\
\text { estudos com cáes assistentes da } \\
\text { terapia. }\end{array}$ \\
\hline
\end{tabular}

\section{Discussáo}

A produção de conhecimento nacional sobre a terapia ocupacional assistida por cães fornece respostas apenas para as perguntas investigativas relativas à populaçáo focalizada neste tipo de terapia, os respectivos objetivos e resultados terapêuticos alcançados.

No próprio estudo de revisão em que se obteve essas respostas, em Mandrá et al. (2019), também apenas um número pequeno de publicaçôes da terapia ocupacional focalizava o tema. Entretanto, dentre os achados, tanto a população como os resultados terapêuticos foram diversos. Nesta direçáo, internacionalmente, há vários estudos sobre práticas da terapia ocupacional assistidas por cães também com diferentes populaçôes e referindo contribuição positiva para diversos objetivos terapêuticos (Shue et al., 2018; Pietruch et al., 2017; Berget \& Braastad, 2011; Velde et al., 2005; Buckley, 1999; Alfano, 1998; Casey, 1996; Darrah, 1996). Especificamente na área pediátrica, estudos indicam que a intervenção da Terapia Ocupacional Assistida por Cáes favoreceu o 
aumento da participação (Bruce et al., 2015; Solomon, 2010; Sams et al. 2006), melhora nas habilidades motoras grossas (Elmaci \& Cevizci, 2015; Gaeta, 2005), nas habilidades motoras finas (Roehm, 2010), na comunicação verbal e não verbal (Solomon, 2010; Elmaci \& Cevizci, 2015; Amuso, 2003) e nas habilidades psicossociais (Elmaci \& Cevizci, 2015).

O documento da Associação Americana de Terapeutas Ocupacionais (AOTA), que tem por finalidade orientar sobre a estrutura da prática da terapia ocupacional, definindo ocupaçóes e papéis ocupacionais que podem ser alvo desta prática, observa que dentre as atividades diárias de uma pessoa pode existir o cuidar de animais (American Occupational Therapy Association, 2015). Cuidar de animais é, portanto, reconhecido enquanto uma ocupação, na qual se atribui o papel ocupacional de cuidador àqueles que a exercem.

Outros textos da AOTA também atestam que há décadas os animais têm feito parte das intervenções em serviços de saúde. Tais documentos versam sobre o uso da TAA e de cães de assistência na prática da terapia ocupacional e visam a padronizar a terminologia a ser adotada pela profissão ao tratar da TAA e dos cães de assistência, além de referir a qualificação necessária para praticar a TAA e os passos para sua implementaçáo (American Occupational Therapy Association, 2020).

Entretanto, apesar do campo da TAA ter aumentado e de terapeutas ocupacionais terem passado a realizar esta prática, ainda é necessário esclarecer sobre a efetividade deste tipo de intervençâo na vida das pessoas, por meio de evidências cientificamente fundamentadas (Fine et al., 2019). A aplicação dos resultados das pesquisas sobre programas terapêuticos que envolvem animais ainda está em estágios iniciais, sendo recente a integração entre profissionais que praticam a TAA e pesquisadores deste tipo de prática (McCune et al., 2014).

Portanto, os estudos examinados nesta revisão não forneceram respostas para a pergunta investigativa sobre a forma como o cão atuou para auxiliar neste processo, mas este questionamento também persiste na literatura internacional, apesar do significativo quantitativo de publicaçóes.

Nesta direção, Fine et al. (2019) referem que a metodologia da TAA constitui uma questão que precisa ser melhor compreendida, para que se estabeleça, por exemplo, de que forma o cáo atua para auxiliar no processo de reabilitaçáo, assim como o que pode ser feito nas intervençôes e de que maneira. Assim, a utilização, o registro e a divulgação de métodos confiáveis são necessários. Isto inclui, por exemplo, a coleta das informaçôes com o registro padronizado de dados comportamentais, emocionais e fisiológicos, tanto do animal como do ser humano. A padronização da avaliação das práticas de TAA permitirá o agrupamento dos resultados cientificamente produzidos, que podem entáo ser comparados com resultados de outros estudos e/ou reaplicados (Fine et al., 2019).

Recentemente, Hill et al. (2019) publicaram um protocolo de intervenção terapêutica ocupacional assistida por cáes para crianças com transtorno do espectro autista (TEA). Na sequência, Hill et al. (2020a), em um estudo controlado e randomizado, analisaram o efeito deste protocolo em comparação às intervençóes consideradas convencionais para esta população. O protocolo elaborado e publicado por Hill et al. (2019) é essencial, pois, apesar da crescente produção de conhecimento relativo ao impacto positivo da terapia assistida por animais para crianças com TEA, 
ainda não existem padróes universais ou diretrizes formalizadas para este tipo de prática pela terapia ocupacional.

Nesta direção, a aplicação e testagem do protocolo obteve a confirmação de que a incorporaçáo de um cão de terapia em sessóes de terapia ocupacional aumentou a quantidade de tempo de envolvimento da criança nas tarefas previstas na sessão e possibilitou a concretização dos objetivos e metas terapêuticas em maior frequência em comparação com as sessôes de terapia ocupacional sem cães (Hill et al., 2019, 2020a). As autoras, mesmo diante dos resultados positivos obtidos, reforçam a necessidade de pesquisas futuras para obtençáo de respostas relativas à formação do terapeuta ocupacional para o exercício da TAA, o treinamento requerido ao cão para que forneça assistência à terapia, assim como os riscos de se incluir um cáo nas sessóes de terapia ocupacional, entre outros aspectos, que são essenciais para a garantia da efetividade da terapia, segurança e bem-estar dos sujeitos-alvo da intervenção e do cão de terapia envolvido (Hill et al., 2020a).

Apesar dos estudos analisados por esta revisão não fornecerem informaçôes sobre a formação do terapeuta ocupacional e o treinamento que o cão necessita para a realização das sessóes de terapia, outro estudo recente de Hill et al. (2020b), sobre os desafios para a prática da terapia ocupacional assistida por cães, traz proposições para a composição de conhecimento desta área.

Dentre as proposiçôes de Hill et al. (2020b), a incorporação de um cão nas sessóes, pelos terapeutas ocupacionais, deve ser pautada pela realização de uma intervenção baseada em evidências e centrada na ocupaçáo. $\mathrm{O}$ cão é, portanto, compreendido como um elemento novo e potencialmente motivador para a concretização das sessôes, sendo que a sua participação na terapia deve estar em consonância com o objetivo terapêutico estabelecido, ou seja, com a demanda referida pelo sujeito-alvo da intervenção. $O$ terapeuta ocupacional deve ser capaz de dividir sua atenção entre a criança e o cão assistente, enquanto mantém o foco no objetivo terapêutico e na eficácia da intervenção. O cão poderá ter uma participação ativa ou passiva nas tarefas terapêuticas, sendo primeiro necessário que o terapeuta ocupacional tenha o conhecimento sobre de que forma o cão poderá dar assistência à terapia e facilitar a conquista dos objetivos terapêuticos. Para isso, o terapeuta ocupacional precisa ter uma formação teórica e prática em TAA e o cáo precisa ter temperamento e comportamento previsível e confiável, tendo recebido treinamento de obediência e sido socializado no ambiente clínico. O cão de terapia também precisa passar por avaliação veterinária periódica de saúde física e emocional, assim como realização de vacinação anual e tratamento preventivo de pulgas, carrapatos e parasitas (Hill et al., 2020b).

$\mathrm{Na}$ tentativa de ampliar o conhecimento sobre a formação dos terapeutas e treinamento dos cães, e também responder às questóes investigativas da presente revisão, destaca-se o modelo piramidal de competências dos profissionais em três níveis (essenciais, intermediárias, profissionais) de Stewart (2014), que também fornece informações sobre o conjunto de conhecimentos, habilidades e atitudes necessários.

Em resumo, o conhecimento demandado abrange saber sobre o animal (raça, espécie, fisiologia, comportamento e histórico) e o bem-estar, cuidados e saúde do animal, e sobre os princípios específicos da TAA articulados à área de atuação do profissional. Em conjunto, é necessário o conhecimento sobre métodos e técnicas de treinamento positivo e não coercitivo para treinar e socializar os animais para uma variedade de ambientes e 
situações, bem como para educar outras pessoas sobre como interagir com o animal. Por fim, o profissional deverá possuir conhecimento baseado em evidências sobre o histórico e tipos de TAA, como os animais podem ser utilizados em ambientes terapêuticos, sobre o vínculo humano-animal, o impacto fisiológico e neurológico da interação entre o ser humano e animal, indicaçóes, contraindicações e formas de gerenciamento de riscos, entre outros (Stewart, 2014; Pet Partners, 2019).

No que tange às habilidades, são necessárias as de prevenção do estresse e esgotamento animal, por meio do suprimento das necessidades do animal, de descanso, hidratação, nutrição e evacuação. Também é preciso desenvolver habilidades para avaliar de forma objetiva a adequação de um animal à terapia e à população-alvo, bem como os pontos fortes e limitaçóes do animal, além de identificar possíveis preconceitos pessoais e/ou culturais em relação ao(s) animal(is) da terapia. Essas habilidades são, portanto, concernentes à incorporação intencional da TAA no plano e processo terapêutico de uma pessoa, mediante a avaliação e seleção das estratégias e açôes apropriadas para cada contexto, com base em cada sessão e nos objetivos do tratamento, com constante avaliação dos resultados e redefinição dos objetivos terapêuticos. O profissional deverá ser hábil não só em sua área de especialidade como também no atendimento e cuidado das pessoas e dos animais simultaneamente, assim como no impacto da terapia no paciente e no animal (Stewart, 2014; Pet Partners, 2019).

Em relação às atitudes priorizadas, está a total responsabilidade do profissional para com o(s) animal(is) envolvido(s) na terapia em termos de bem-estar, defesa, segurança e respeito às características, interesses e disposição do animal em participar. $\mathrm{O}$ mesmo tipo de responsabilidade é válido para com os sujeitos alvo da intervenção. Em conjunto, cabe ao profissional promover a conscientização, educação e formação sobre a TAA nos níveis micro e macro (individual, comunidade, público), manter-se familiarizado com a literatura existente, adotar a linguagem e terminologia atual e apropriada e atuar no desenvolvimento contínuo da literatura da TAA (Stewart, 2014; Pet Partners, 2019).

\section{Consideraçóes Finais}

A presente revisão de escopo não encontrou estudos da terapia ocupacional tendo cães como assistentes das intervençôes no Brasil. Dentre os cinco estudos encontrados e analisados nesta revisão, um foi uma revisão que referiu a terapia ocupacional como uma das profissóes que, no contexto internacional, realiza a terapia assistida por cães com pessoas com deficiência e/ou sequelas físicas ou mentais, sendo os objetivos e resultados terapêuticos de reabilitação física ou cognitiva. Os demais estudos se referiam ao cuidar de animais compondo uma das ocupações humanas e um dos papéis ocupacionais que as pessoas podem assumir. $\mathrm{O}$ próprio documento relativo à estrutura da prática da profissão inclui o cuidar de animais enquanto uma ocupação e papel ocupacional, podendo estes serem alvo das intervençóes da terapia ocupacional.

Embora não tenham sido identificadas publicaçôes nacionais capazes de elucidar todas as questôes investigativas, há uma literatura internacional que traz informaçóes esclarecedoras sobre a formação e as competências necessárias ao terapeuta e o treinamento requerido ao cão assistente da terapia ocupacional.

A incipiente produção nacional indica a necessidade de pesquisas futuras sobre o tema, especificamente a sistematização das práticas baseadas em evidências para a 
compreensão das demandas da população-alvo, dos objetivos terapêuticos ocupacionais e a(s) forma(s) de concretizá-los, tendo o cão como assistente. Em adição, há a demanda por novas pesquisas sobre a formação e competências do profissional terapeuta ocupacional para a prática da TAA e sobre o treinamento do cão, de forma a fomentar práticas terapêuticas eficazes e cientificamente embasadas. A literatura internacional indica a necessidade de investigaçóes sobre a forma como o cáo pode atuar para auxiliar no processo de reabilitação, sendo necessário para isso o desenvolvimento de medidas de análise e de avaliação precisas e passíveis de replicação em outras intervençóes. Por fim, por ser a primeira revisão de escopo nacional sobre um tema, com um número restrito de publicaçóes, este estudo possui limitaçóes na possibilidade de generalização, devendo ser considerado sob essa perspectiva.

\section{Referências}

Abate, S. V., Zucconi, M., \& Boxer, B. A. (2011). Impact of canine-assisted ambulation on hospitalized chronic heart failure patients' ambulation outcomes and satisfaction: a pilot study. The Journal of Cardiovascular Nursing, 26(3), 224-230. http://dx.doi.org/10.1097/JCN.0b013e3182010bd6.

Alfano, T. R. (1998). A survey of the use of animal-assisted therapy as a modality in the field of occupational therapy (Master's thesis). D'Youville College, Buffalo, New York.

American Occupational Therapy Association - AOTA. (2015). Estrutura da prática da terapia ocupacional: domínio \& processo. Revista de Terapia Ocupacional da Universidade de São Paulo, 26(Esp), $1-49$.

American Occupational Therapy Association - AOTA. (2020). Incorporating animal assisted therapy and assistance dogs into practice. Bethesda: AOTA Press. Recuperado em 25 de abril de 2020, de https://www.aota.org/Practice/RehabilitationDisability/serviceanimalassistedtherapy.aspx

Amuso, S. A. (2003). The effect animal assisted therapy has on the spontaneous interaction of autistic andlor pervasively developmentally delayed children (Master's thesis). Touro College, New York.

Arksey, H., \& O’Malley, L. (2005). Scoping studies: towards a methodological framework. International Journal of Social Research Methodology, 8(1), 19-32.

Barker, S. B., Knisely, J. S., McCain, N. L., \& Best, A. M. (2005). Measuring stress and immune response in healthcare professionals following interaction with a therapy dog: a pilot study. Psychological Reports, 96(3 Pt 1), 713-729. http://dx.doi.org/10.2466/pr0.96.3.713-729.

Berget, B., \& Braastad, B. O. (2011). Animal-assisted therapy with farm animals for persons with psychiatric disorders. Annali dell'Istituto Superiore di Sanita, 47(4), 384-390.

Braun, C., Stangler, T., Narveson, J., \& Pettingell, S. (2009). Animal-assisted therapy as a pain relief intervention for children. Complementary Therapies in Clinical Practice, 15(2), $105-$ 109

Bruce, S. M., Feinstein, J. D., Kennedy, M. C., \& Liu, M. (2015). Humane education for students with visual impairments: learning about working dogs. Journal of Visual Impairment \& Blindness, 109(4), 281-290.

Buckley, L. S. (1999). Occupational therapists' interests and attitudes toward animal assisted therapy as treatment modality (Master's thesis). Florida International University, Miami.

Camp, M. M. (2001). The use of service dogs as an adaptive strategy: a qualitative study. The American Occupational Therapy Association, 55(5), 509-517.

http://dx.doi.org/10.5014/ajot.55.5.509. 
Casey, H. M. (1996). A survey of occupational therapists using pet-facilitated therapy. Journal of Home Health Care Practice, 8(4), 10-17.

Centro Latino-Americano e do Caribe de Informação em Ciências da Saúde - BIREME. (2018). Recuperado em 25 de abril de 2020, de http://decs.bvs.br/P/decsweb2020.htm

Cid, M. F., Santos, G., \& Squassoni, C. (2017). Cotidiano e práticas educativas parentais: a percepção das famílias de crianças em sofrimento psíquico. Revista de Terapia Ocupacional da Universidade de São Paulo, 28(2), 190-197.

Cole, K. M., Gawlinski, A., Steers, N., \& Kotlerman, J. (2007). Animal-assisted therapy in patients hospitalized with heart failure. American Journal of Critical Care, 16(6), 575-588.

Colquhoun, H. L., Levac, D., O’Brien, K. K., Straus, S., Tricco, A. C., Perrier, L., Kastner, M., \& Moher, D. (2014). Scoping reviews: time for clarity in definition, methods, and reporting. Journal of Clinical Epidemiology, 67(12), 1291-1294.

Darrah, J. P. (1996). A pilot survey of animal-facilitated therapy in Southern California and South Dakota nursing homes. Occupational Therapy International, 3(2), 105-121.

Davis, B. W., Nattrass, K., O’Brien, S., Patronek, G., \& MacCollin, M. (2004). Assistance dog placement in the pediatric population: Benefits, risks, and recommendations for future application. Anthrozoos, 17(2), 130-145.

Dias, E., Duarte, Y., Morgani, M., \& Lebrão, M. (2014). As Atividades avançadas de vida diária como componente da avaliação funcional do idoso. Revista de Terapia Ocupacional da Universidade de Sáo Paulo, 25(3), 225-232.

Elmaci, D. T., \& Cevizci, S. (2015). Dog-assisted therapies and activities in rehabilitation of children with cerebral palsy and physical and mental disabilities. International Journal of Environmental Research and Public Health, 12(5), 5046-5060.

Fairman, S. K., \& Huebner, R. A. (2001). Service dogs: a compensatory resource to improve function. Occupational Therapy in Health Care, 13(2), 41-52.

Fine, A. H., Tedechi, P., \& Elvove, E. (2019). Forward thinking: the evolving field of humananimal interactions. In A. Fine (Ed.), The handbook of animal assisted therapy (pp. 23-41). Cambridge: Academic Press.

Friedmann, E., Galik, E., Thomas, S. A., Hall, P. S., Chung, S. Y., \& McCune, S. (2015b). Evaluation of a pet-assisted living intervention for improving functional status in assisted living residents with mild to moderate cognitive impairment: a pilot study. American Journal of Alzheimer's Disease and Other Dementias, 30(3), 276-289.

Friedmann, E., Son, H., \& Saleem, M. (2015a). The animal-human bond: health and wellness. In A. Fine (Ed.), Handbook on animal-assisted therapy: theoretical foundations and guidelines for practice (pp. 73-84). New York: Academic Press.

Gaeta, C. A. (2005). The effects of animal assisted therapy on arm reaching movements of children diagnosed with a movement disorder (Doctoral dissertation). D’Youville College, Buffalo.

Hall, S. S., MacMichael, J., Turner, A., \& Mills, D. S. (2017). A survey of the impact of owning a service dog on quality of life for individuals with physical and hearing disability: a pilot study. Health and Quality of Life Outcomes, 15(1), 59.

Hansen, K. M., Messinger, C. J., Baun, M. M., \& Megel, M. (1999). Companion animals alleviating distress in children. Anthrozoos, 12(3), 142-148. http://dx.doi.org/10.2752/089279399787000264.

Herlache-Pretzer, E., Winkle, M. Y., Csatari, R., Kolanowski, A., Londry, A., \& Dawson, R. (2017). The impact of service dogs on engagement in occupation among females with mobility impairments: a qualitative descriptive study. International Journal of Environmental Research and Public Health, 14(6), 649. http://dx.doi.org/10.3390/ijerph14060649.

Hill, J., Ziviani, J., Cawdell-Smith, J., \& Driscoll, C. (2019). Canine assisted occupational therapy: protocol of a pilot randomised control trial for children on the autism spectrum. Open Journal of Pediatrics, 9(3), 199-217. http://dx.doi.org/10.4236/ojped.2019.93020. 
Hill, J., Ziviani, J., Driscoll, C., \& Cawdell-Smith, J. (2020b). Canine-assisted occupational therapy for children on the autism spectrum: challenges in practice. British Journal of Occupational Therapy, 83(4), 215-219. http://dx.doi.org/10.1177/0308022619858851.

Hill, J., Ziviani, J., Driscoll, C., Teoh, A. L., Chua, J. M., \& Cawdell-Smith, J. (2020a). Canine assisted occupational therapy for children on the autism spectrum: a pilot randomised control trial. Journal of Autism and Developmental Disorders, 50(11), 4106-4120. http://dx.doi.org/10.1007/s10803-020-04483-7.

International Association of Human-Animal Interaction Organizations - IAHIO. (2018). The IAHAIO definitions for animal-assisted intervention and guidelines for wellness of animals involved. Recuperado em 25 de abril de 2020, de https://iahaio.org/wp/wpcontent/uploads/2018/04/iahaio_wp_updated-2018-final.pdf

Isaacson, M. (2013). The training and use of service dogs in occupational therapy education. The Open Journal of Occupational Therapy, 1(2), 1-13.

Kruger, K. A., \& Serpell, J. A. (2006). Animal-Assisted interventions in mental health: definitions and theoretical foundations. In A. Fine (Ed.), Handbook on animal-assisted therapy: theoretical foundations and guidelines for practice (pp. 21-38). New York: Academic Press.

LaJoie, K. R. (2003). An evaluation of the effectiveness of using animals in therapy (Doctor's thesis). Spalding University, Louisville.

Lopes, P., \& Matsukura, T. (2018). Perspectivas futuras sobre participação social na ótica de adolescentes com paralisia cerebral e suas mães. Revista de Terapia Ocupacional da Universidade de São Paulo, 29(1), 19-26.

Mandrá, P. P., Moretti, T. C. F., Avezum, L. A., \& Kuroishi, R. C. S. (2019). Terapia assistida por animais: revisão sistemática da literatura. CoDAS, 31(3), e20180243. http://dx.doi.org/10.1590/2317-1782/20182018243.

Martin, F., \& Farnum, J. (2002). Animal-assisted therapy for children with pervasive developmental disorders. Western Journal of Nursing Research, 24(6), 657-670.

McCune, S., Kruger, K. A., Griffin, J. A., Esposito, L., Freund, L. S., Hurley, K. J., \& Bures, R. (2014). Evolution of research into the mutual benefits of human-animal interaction. Animal Frontiers, 4(3), 49-58.

Motooka, M., Koike, H., Yokoyama, T., \& Kennedy, N. L. (2006). Effect of dog-walking on autonomic nervous activity in senior citizens. The Medical Journal of Australia, 184(2), 6063.

Nagengast, S. L., Baun, M. M., Megel, M., \& Leibowitz, J. M. (1997). The effects of the presence of a companion animal on physiological arousal and behavioral distress in chilldren during a physical examination. Journal of Pediatric Nursing, 12(6), 323-330.

Nordgren, L., \& Engstrom, G. (2012). Effects of animal-assisted therapy on behavioral and/or psychological symptoms in dementia: a case report. American Journal of Alzheimer's Disease and Other Dementias, 27(8), 625-632.

O’Brien, K. K., Colquhoun, H., Levac, D., Baxter, L., Tricco, A. C., Straus, S., Wickerson, L., Nayar, A., Moher, D., \& O’Malley, L. (2016). Advancing scoping study methodology: a web-based survey and consultation of perceptions on terminology, definition and methodological steps. BMC Health Services Research, 26(16), 305.

Orlandi, M., Trangeled, K., Mambrini, A., Tagliani, M., Ferrarini, A., Zanetti, L., Tartarini, R., Pacetti, P., \& Cantore, M. (2007). Pet therapy effects on oncological day hospital patients undergoing chemotherapy treatment. Anticancer Research, 27(6C), 4301-4303.

Pet Partners. (2019). Animal-assisted interventions competencies. Recuperado em 25 de abril de 2020, de https://petpartners.org/wp-content/uploads/2014/12/Tiered-AAI-

Competencies_2016.pdf 
Peters, M. D., Godfrey, C. M., Khalil, H., McInerney, P., Parker, D., \& Soares, C. B. (2015). Guidance for conducting systematic scoping reviews. International Journal of Evidence-Based Healthcare, 13(3), 141-146.

Pietruch, A., Marsh, A., Steixner, B., Forkey, K., \& McFarland, S. (2017). The supports and barriers to the use of animal-assisted therapy in occupational therapy intervention with adults (Master's thesis). Utica College, Utica.

Reed, R., Ferrer, L., \& Villegas, N. (2012). Curadores naturais: uma revisão da terapia e atividades assistidas por animais como tratamento complementar de doenças crônicas. Revista Latino-Americana de Enfermagem, 20(3), 612-618. http://dx.doi.org/10.1590/S010411692012000300025.

Roehm, J. (2010). Animal assisted therapy increases fine motor skills (Master's thesis). Texas Woman's University, Denton.

Şahin, S., Kose, B., \& Zarif, M. (2018). Animal-assisted therapy in occupational therapy. In M. Huri (Ed.), Occupational therapy: therapeutic and creative use of activity (pp. 91-106). London: IntechOpen.

Sams, M. J., Fortney, E. V., \& Willenbring, S. (2006). Occupational therapy incorporating animals for children with autism: a pilot investigation. The American Journal of Occupational Therapy, 60(3), 268-274.

Shue, S. J., Winkle, M. Y., \& Mulcahey, M. J. (2018). Integration of animal-assisted therapy standards in pediatric occupational therapy. People and animals. Internacional Journal of Research and Practice, 1(1), 1-9.

Smyth, C., \& Slevin, E. (2010). Experiences of family life with an autism assistance dog. Learning Disability Practice, 13(4), 12-17.

Solomon, O. (2010). What a dog can do: children with autism and therapy dogs in social interaction. Ethos: Journal of the Society for Psychological Antropology, 38(1), 143-166.

Squilasse, A. F., \& Squilasse Junior, F. T. (2018). Intervençôes assistidas por animais: consideraçôes gerais. Revista de Educação Continuada em Medicina Veterinária e Zootecnia do CRMV-SP, 16(2), 30-35.

Stewart, L. A. (2014). Competencies in animal assisted therapy in counseling: a qualitative investigation of the knowledge, skills and attitudes required of competent animal assisted therapy practitioners (Doctoral dissertation). Georgia State University, Atlanta.

Stumpf, E., \& Breitenbach, E. (2014). Dolphin-assisted therapy with parental involvement for children with severe disabilities: further evidence for a family-centered theory for Effectiveness. Anthrozoos, 27(1), 95-109. http://dx.doi.org/10.2752/175303714X13837396326495.

Tricco, A. C., Lillie, E., Zarin, W., O’Brien, K., Colquhoun, H., Kastner, M., Levac, D., Sharpe, J. P., Wilson, K., Kenny, M., Warren, R., Wilson, C., Stelfox, H. T., \& Straus, S. E. (2016). A scoping review on the conduct and reporting of scoping reviews. BMC Medical Research Methodology, 16, 1-10. http://dx.doi.org/10.1186/s12874-016-0116-4.

Tsai, C. C., Friedmann, E., \& Thomas, S. A. (2010). The effect of animal-assisted therapy on stress responses in hospitalized children. Anthrozoos a Multidisciplinary Journal of the Interactions of People \& Animals, 23(3), 245-258. http://dx.doi.org/10.2752/175303710X12750451258977.

VanFleet, R., Fine, A., Oçallaghan, D., Mackintosh, T., \& Gimeno, J. (2019). Application of animal-assisted interventions in professional settings: an overview of alternatives. In A. Fine (Ed.), The handbook of animal assisted therapy (pp. 158-174). Cambridge: Academic Press.

Velde, B. P., Cipriani, J., \& Fisher, G. (2005). Resident and therapist views of animal-assisted therapy: implications for occupational therapy practice. Australian Occupational Therapy Journal, 52(1), 43-50. 
Viau, R., Arsenault-Lapierre, G., Fecteau, S., Champagne, N., Walker, C. D., \& Lupien, S. (2010). Effect of service dogs on salivary cortisol secretion in autistic children.

Psychoneuroendocrinology, 35(8), 1187-1193.

\section{Contribuiçáo dos Autores}

Mirela de Oliveira Figueiredo: contribuiu com a

concepção teórica e metodológica do estudo, coleta de dados, discussão e redação. Ana Luiza Alegretti e Lilian Magalhães: contribuíram no aporte teórico e metodológico e revisão final. Todos os autores aprovaram a versão final do texto.

\section{Fonte de Financiamento}

FAPESP processo no 2019/10680-5.

\section{Autor para correspondência}

Mirela de Oliveira Figueiredo

e-mail: mirelafigueiredo@gmail.com

\section{Editora de seçáo}

Profa. Dra. Marcia Maria Pires Camargo Novelli 